\title{
El género Deguelia (Leguminosae, Papilionoideae, Millettieae) en Mesoamérica, una especie nueva y una combinación nueva
}

\author{
The genus Deguelia (Leguminosae, Papilionoideae, Millettieae) in Mesoamerica, \\ a new species and a new combination
}

\author{
Mario Sousa S. \\ Departamento de Botánica, Instituto de Biología, Universidad Nacional Autónoma de México, Apartado postal 70-367, 04510 México, D. F., México. \\ Correspondencia: sousa@servidor.unam.mx
}

\begin{abstract}
Resumen. Se presenta una revisión taxonómica del género Deguelia Aubl. (Millettieae: Leguminosae) para Mesoamérica; para ello fue necesario describir e ilustrar a una nueva especie, Deguelia alata M. Sousa y proponer una nueva combinación, D. densiflora (Benth.). A. M. G. Azevedo ex M. Sousa, para una especie previamente incluida en el género Lonchocarpus.

Palabras clave: Deguelia, Leguminosae, Colombia, Costa Rica, Guatemala, Nicaragua, Panamá.

Abstract. A revision of the genus Deguelia Aubl. (Millettieae: Leguminosae) in Mesoamerica is presented. A new species, Deguelia alata is described and illustrated, and a new combination, D. densiflora is proposed for a species formerly included in Lonchocarpus.
\end{abstract}

Key words: Deguelia, Leguminosae, Colombia, Costa Rica, Guatemala, Nicaragua, Panama.

\section{Introducción}

Este trabajo se enmarca dentro del Proyecto Flora Mesoamericana, el formato del cual no permite efectuar cambios o formalizaciones nomenclaturales y limita la cita de ejemplares y su información.

El género Deguelia fue descrito por Aublet (1775), restablecido por Geesink (1984) y adoptado por Azevedo-Tozzi (1989, 1994, 1998). Deguelia se localiza fundamentalmente en las áreas selváticas de Sudamérica y marginalmente en Centroamérica, y lo representan alrededor de 16 especies (Azevedo-Tozzi 1989, 1994, 1998).

Deguelia ha sido considerado parte de Lonchocarpus, Bentham (1860) y Pittier (1917), o bien de Derris (véase Mattos, 1988), incluyendo tanto a Deguelia como a Lonchocarpus, o sólo las especies de bejucos leñosos o arbustos y árboles de Lonchocarpus con frutos alados como Derris, Poppendieck (1999). En la actualidad, Derris se considera un género de los trópicos de África oriental, del sur de Asia y de Australia.

Las especies aquí tratadas se ubican en Deguelia y no

Recibido: 02 junio 2008; aceptado: 04 noviembre 2008 en Lonchocarpus, ya que son bejucos leñosos, de hojas con presencia de estipelas; inflorescencias en pedúnculos florales (braquiblastos) multifloros ensanchados y callosos; estambre vexilar libre o casi libre, sí unido sólo en la base, donde se presentan 2 fenestras sin margen calloso; y la presencia de un anillo intraestaminal rodeando la base del ovario.

En el área Mesoamericana sólo se han localizado 2 especies, de las que una requiere ser trasferida del género Lonchocarpus a Deguelia y la otra resultó ser nueva para la ciencia.

\section{Descripciones}

Deguelia Aubl.

Bejucos leñosos. Hojas alternas, con arreglo helicoidal en el tallo, imparipinnadas, frecuentemente congestas en brotes cortos, 5-13-folioladas; raquis foliar frecuentemente con estipelas aciculares, caducas, folíolos opuestos, los márgenes enteros, aplanados a ondulados. Inflorescencias generalmente largas, formadas de cimas racemosas, cuyos pedúnculos florales (braquiblastos) en ocasiones son alargados (los proximales), delgados y/o del tercio inferior al ápice reducidos, engrosados, congestifloros, 
fasciculados; bractéolas en pares, cercanas o distantes del cáliz; cáliz dentado en ocasiones giboso en la base, frecuentemente con desarrollo de hipanto; pétalos seríceos a casi glabros, a menudo con líneas y puntos translúcidos, la lámina del estandarte suborbicular, oblonga, oblata, con o sin aurículas en la base, con o sin áreas callosas, las alas ligeramente adheridas a la quilla; alas y quilla en ocasiones fuertemente arqueadas, generalmente rectas; estambre vexilar totalmente libre (diadelfo) o sólo unido en la base (seudomonadelfo) formando 2 fenestras sin margen calloso; anteras uniformes; anillo intraestaminal basal en ocasiones presente rodeando al ovario; ovario 2-18-ovulado. Legumbre generalmente indehiscente, en ocasiones dehiscente, lomentácea, drupácea, aplanada, membranácea a leñosa, con o sin desarrollo del margen vexilar en un ala; semillas con el hilo lateral a subterminal.

Clave para las especies mesoamericanas:

1a. Hojas 5-folioladas; corola blanca; pétalos de alas y quilla rectos no arqueados; ovario 3-ovulado; legumbre oblongo-elíptica, cartácea, aplanada, alada; semillas ca. $21 \mathrm{~mm}$ de largo. 1. D. alata

1b. Hojas 7-11-folioladas; corola rosada a púrpura, pétalos de alas y quilla fuertemente arqueados; ovario 10-18-ovulado; legumbre linear, leñosa, lomentácea, sin ala; semillas 9.5-10 mm de largo...... 2. D. densiflora

1. Deguelia alata M. Sousa, sp. nov. Tipo: Colombia. Atlántico en el límite con el Departamento Bolívar, Los Pendales, hacienda Riodulce, altitud 10-20 m; bosques espesos, A. Dugard y R. Jaramillo 4156 ( holotipo: MEXU; isotipos, COL, NY). Fig. 1.

Deguelia utilis in part sensu A. M. G. Azevedo non Lonchocarpus utilis A. C. Sm.

A Deguelia amazonica (Killip) Killip differt pilositate praecipue fusco-luteola (vs. praecipue ferruginea), foliis 5-foliolatis (vs. 7-9-foliolatis), marginibus foliolorum aliquantum undulatis (vs. applanatis), inflorescentiis tantum pedunculis brevibus incrassatis, congestifloris (vs. pedunculis floralibus proximalibus elongatis, gracilibus, laxifloris), pedicellis 1.5-3 mm longis (vs. 4-7 mm), bracteolis proximalis ad calycem adpressis (vs. bracteolis a calycis base remotis), corolla alba (vs. lilacina vel purpurea), pilosa (vs. glabra), lamina vexilli sine callo basali (vs. lamina vexilli base callosa), abaxialiter pilositate canescente (vs. abaxialiter glabra).

Bejucos leñosos en la copa de los árboles; corteza interior con fluido resinoso rojizo al corte; ramas jóvenes canescentes a pardo-amarillentas seríceas a tomentulosas, pronto glabrescentes. Hojas con arreglo helicoidal en el tallo, frecuentemente congestas en brotes cortos; estípulas no vistas; pecíolo $6.5-11 \mathrm{~cm}$ de largo, canaliculado, hojas 5-folioladas; raquis foliar con estipelas ca. $6.3 \mathrm{~mm}$ de largo, aciculares, caducas; folíolos 7.5-11(-12.5) cm de largo, 4.5-6(-7.5) cm de ancho, ovados a elíptico-ovados, cartáceos a subcoriáceos, epunteados, la base redondeada, los márgenes ondulados, el ápice corto-acuminado, el haz ligeramente brillante, glabrescente a glabro, el envés moderada a esparcidamente pardo-amarillento seríceo; nervaduras primaria y secundarias algo realzadas en el envés, las laterales 6-8. Inflorescencias (6-)14-24.5 cm de largo, erectas, pedunculadas, los pedúnculos 1-2.5 cm de largo, en brotes cortos, axilares, simples; floración coetánea; pedúnculos florales (braquiblastos) 1.5-2.5(-4.5) $\mathrm{mm}$ de largo, engrosados, congestifloros, irregularmente asimétricos, con 6-7 flores; pedicelos 1.5-3 mm de largo, delgados; bractéolas 0.9-1 mm de largo, suborbiculares, ovadas, aplicadas en la base del cáliz, persistentes. Botones florales elipsoidales. Flores 11-12 mm de largo; cáliz 4-5 $\mathrm{mm}$ de largo, ciatiforme, dentado, el diente carinal ca. 1 $\mathrm{mm}$ de largo, triangular, agudo, esparcidamente punteado, densamente pardo-amarillento seríceo; corola blanca, moderada a densamente canescente a pardo-amarillenta serícea, con líneas y puntos translúcidos; lámina del estandarte 7.7-8 mm de ancho, suborbicular, recta, el ápice casi entero abaxialmente, con aurículas muy reducidas, sin apéndices callosos en la base, redondeada, canescentepelosa en la parte central de la base que se continúa a lo largo de la uña, la uña ca. 2.6 mm de largo, delgada; alas y quilla rectas; ovario 3-ovulado, anillo presente, rodeando la base del ovario. Legumbre 7.2-10.5 cm de largo, 2.7-3 cm de ancho, oblongo-elíptica, indehiscente, la base obtusa, algo asimétrica, el ápice obtuso cortamente rostrado, aplanada, cartácea, moderada a esparcidamente pardo-amarillenta, serícea a tomentulosa, el margen vexilar alado, el ala hasta ca. $6 \mathrm{~mm}$ de ancho; semillas 2-3, ca. $21 \mathrm{~mm}$ de largo, ca. $11 \mathrm{~mm}$ de ancho, ca. $3.2 \mathrm{~mm}$ de grosor, color castaño.

\section{Comentarios taxonómicos}

Distribución, hábitat y fenología. Conocida sólo de Panamá y Colombia. En Panamá se encuentra en el Darién y la provincia de Panamá; en Colombia en la costa caribeña. En selvas altas perennifolias a la orilla de cuerpos de agua; climas cálido húmedos; del nivel del mar a 100 $\mathrm{m}$ de altitud. La floración se presenta a finales de enero; la fructificación se inicia a mediados de febrero, con frutos maduros presentes a principios de abril.

Material adicional examinado. PANAMÁ: Darién: near 

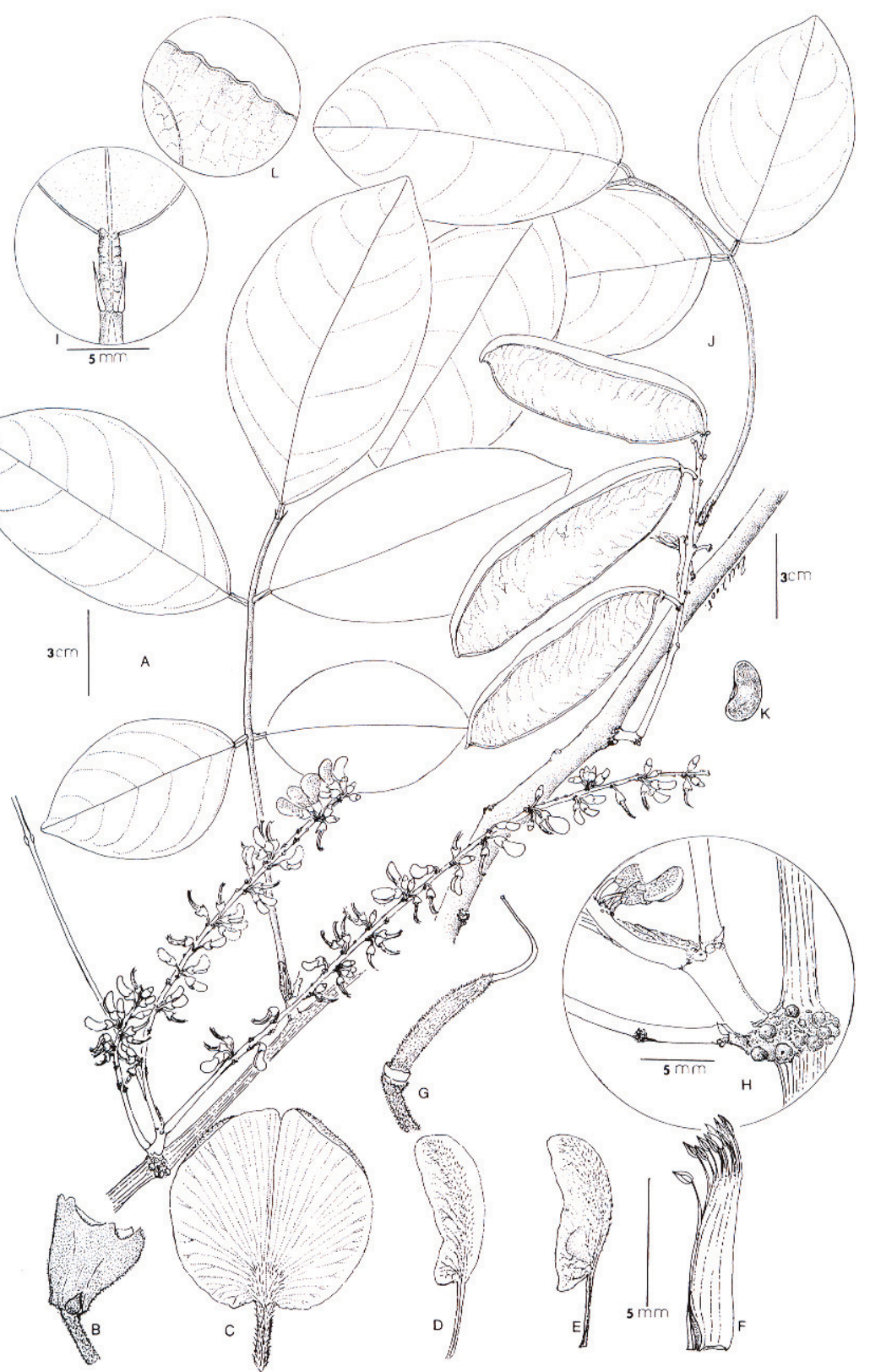

Figura 1. Deguelia alata. a, rama con inflorescencia y hojas; b, cáliz con bractéola y pedicelo; c, estandarte cara abaxial, mostrando lámina y uña; d, ala; e, pétalo de la quilla; f, tubo estaminal, mostrando los estambres y una ventana basal; g, gineceo, mostrando en su base al anillo; $h$, brote corto, mostrando catáfilos y base de inflorescencia y pecíolo; i, estipelas; $\mathrm{j}$, infrutescencias, con frutos alados; $\mathrm{k}$, semilla; 1, margen del folíolo. La rama con inflorescencias, hojas, margen del folíolo, estipelas, brote corto y flor disectada, de Dugand y Jaramillo 4156 (MEXU, tipo), frutos y semilla de Gentry 5045 (F). 
Refugio, 15-21 miles of Santa Fe, J. A. Duke 10288 (MO, NY). Panamá: near archaeological site at edge of Madden Lake, A. Gentry 5045 (F).

La recolecta de Dugard y Jaramillo 4156 (COL, MEXU, NY) fue interpretada por Azevedo-Tozzi (1998) como el material fértil en flor de Deguelia utilis (A. C. Sm.) A. M. G. Azevedo, quien lo designó como epitipo; sin embargo, este ejemplar difiere taxonómicamente del holotipo de Deguelia utilis Killip y A. C. Smith 27278 (US!). Deguelia utilis fue descrita con base en material vegetativo. En el cuadro 1 se comparan ambas especies y se indican 9 caracteres vegetativos que las diferencian. Con base en estas diferencias, aquí se tratan como 2 especies, de las que Deguelia alata es nueva para la ciencia y $D$. utilis permanece sin conocerse el material fértil.

Deguelia alata se relaciona con D. amazonica (Killip) Killip por su legumbre alada, pero diferenciándose de ella por varios caracteres, tanto vegetativos como florales; así, difiere de Deguelia amazonica por su pelosidad en ramas jóvenes, es canescente a pardo amarillenta, serícea a tomentulosa (vs. ferrugínea); hojas 5-folioladas (vs. 79-folioladas); margen foliar ondulado (vs. aplanado); inflorescencia con el pedúnculo breve ensanchado, congestifloro (vs. pedúnculos florales proximales alargados, delgados laxifloros); pedicelos 1.5-3 mm de largo (vs. 4$7 \mathrm{~mm}$ ); bractéolas próximas al cáliz y adpresas a él (vs. bractéolas lejanas al cáliz), corola blanca, serícea (vs. lila a púrpura, glabra); lámina del estandarte sin callos basales (vs. lámina del estandarte con base callosa); superficie adaxial canescente pelosa (vs. glabra). D. amazonica se distribuye en la cuenca del Amazonas, mientras que D. alata habita en Panamá y en la vertiente caribeña de Colombia.

Etimología. El epíteto hace énfasis en la notable ala de la legumbre.
2. Deguelia densiflora (Benth.) A. M. G. Azevedo ex M. Sousa, comb. nov., Lonchocarpus densiflorus Benth. Ann. Nat. Hist. 3: 437. 1839. Tipo: GUYANA, Banks of Rio Essequibo, R. Schomburg 52 (holotipo: K!; isotipos, BM!, $\mathrm{P} !)$.

Lonchocapus glabrescens Benth. in Hook. Kew J. Bot. 2: 233. 1850. Tipo: Brasil, North, Pará. R. Spruce s. $n$. (holotipo: $\mathrm{K}$ !; isotipo, DS!). Ilustración: Bentham, Bentham in Martius, Fl. Bras. 15 (2): f. 106. 1876.

Lonchocarpus boliviensis Pittier, Contr. U. S. Natl. Herb. 20: 93. 1917. Tipo: BolIVIA, junction of Rivers Beni and Madre de Dios, H. H. Rusby 2362 (holotipo: US!; isotipo, $\mathrm{K} !)$

Derris glabrescens (Benth.) J. F. Macbride, Field Mus. Nat. Hist. 13: 261. 1943.

Nombres vulgares. Quiebra hacha, Costa Rica; bastardhiam, Guyana; timbo, barbasco, Brasil.

Bejucos leñosos 3-30 m de alto; corteza interior con fluido resinoso al corte; ramas moderadamente canescentes seríceas, pronto glabrescentes. Hojas con arreglo helicoidal en el tallo; estípulas $2.5-3 \mathrm{~mm}$ de largo, subuladas, caducas; pecíolo $2.5-6.5 \mathrm{~cm}$ de largo, terete acostillado; hojas 7-11-folioladas; raquis foliar con estipelas, éstas aciculares, persistentes; folíolos 3-8 cm de largo, 2-4 cm de ancho, ovados a oblongo-elípticos, coriáceos, epunteados, aunque frecuentemente las áreas entre las nervaduras irregularmente translúcidas, el ápice abruptamente acuminado, el haz brillante y glabro, el envés esparcidamente seríceo; nervaduras primaria y secundarias realzadas en el envés, las nervaduras secundarias 6-13. Inflorescencias $17-38 \mathrm{~cm}$ de largo, largamente pedunculadas; floración tardía; pedúnculos florales (braquiblastos) 2-3.5 mm de largo, multifloros, fasciculados, engrosados, irregularmente claviformes;

Cuadro 1. Comparación de caracteres vegetativos entre Deguelia utilis y D. alata

\begin{tabular}{lcc}
\hline & $D$. utilis & D. alata \\
\hline Pubescencia, color & ferrugínea & pardo-amarillenta \\
Brotes foliáceos & generalmente largos & cortos \\
Pecíolo & terete & presentes, pronto caducas \\
Estipelas & ausentes & sólo 5 \\
Número de folíolos & generalmente 7 & ovados a elíptico-ovados \\
Folíolos, forma & elípticos a angostamente elípticos & aplanada sin ocultar la nervadura media \\
Base del folíolo en el haz & plegada y ocultando la nervadura media & ondulados \\
Márgenes & aplanados & cortamente acuminados \\
Folíolos, ápice & largamente acuminados a caudados &
\end{tabular}


pedicelos 4-9 mm de largo, delgados; bractéolas 1.3-2 $\mathrm{mm}$ de largo, oblongo-orbiculares a oblatas, opuestas, aplicadas al cáliz. Flores 12-13 mm de largo; cáliz 4-4.5 $\mathrm{mm}$ de largo, anchamente campanulado, brevidentado, ferrugíneo seríceo, moderadamente con líneas y puntos translúcidos; corola rosada a púrpura, la lámina del estandarte 10-12 mm de ancho, orbicular a oblata, densamente serícea adaxialmente, con abundantes líneas y puntos translúcidos, base algo auriculada, la uña ca. $1 \mathrm{~mm}$ de largo, callosa, las alas y la quilla fuertemente arqueadas; ovario 10-18-ovulado, anillo intraestaminal presente, rodeando la base del ovario. Legumbre 7-20 cm de largo, $1.3-1.5 \mathrm{~cm}$ de ancho, ca. $0.2 \mathrm{~cm}$ de grosor, indehiscente, lomentácea, fragmentándose en artículos cuadrangulares monospermos, leñosa, linear, casi sésil, rostrada, aplanada, canescente serícea, las suturas aquilladas; semillas 3-12, 9.5-10 mm de largo, pardo-amarillentas.

\section{Comentarios taxonómicos}

Distribución, hábitat y fenología. Ampliamente distribuida en selvas húmedas neotropicales de Centroamérica a Sudamérica. En Mesoamérica, de una sola colecta antigua en Guatemala, y más recientemente de Nicaragua a Panamá en selvas a la orilla de cuerpos de agua. En Sudamérica, en las cuencas de los ríos Orinoco y Amazonas, en áreas inundables, corrientes de agua, lagos, y en ocasiones en áreas sabanoides. La altitud en la que habita va de 0 a $40 \mathrm{~m}$ en Mesoamérica, de 10 a $250 \mathrm{~m}$ en Sudamérica. La floración en Mesoamérica y Colombia, de finales de agosto a finales de noviembre; en Brasil y Perú, de mayo a mediados de septiembre; en Venezuela, de finales de febrero a principios de marzo; fructifica de finales de diciembre a mediados de febrero y hasta finales de marzo.

Especie cuyos frutos maduros se fragmentan en artículos monospermos, que se dispersan por corrientes de agua; tipo de fruto muy característico y único de esta especie.

Material representativo. GUATEMaLA: $E$. $R$. von Friedrichsthal s.n. (W). NICARAGUA: Río San Juan: Reserva Indio-Maíz, a lo largo de la ribera del Río Indio, $R$. Rueda et al. 8833 (MEXU). CosTA RICA: Limón: Refugio Gandoca-Manzanillo, along Río Gandoca from mouth to ca. $2 \mathrm{~km}$ upstream, M. Grayum et al. 8062 (MEXU, MO); Suerre and Dos Bocas, drenajes de los Ríos Parismina and Reventazón, P. J. Shank y A. Molina R. 4291 (F, GH); Cerro Coronel, along Río Colorado at and below outflow of Laguna Colorada at and below outflow of Laguna Dante, W. D. Stevens 24014 (MEXU, MO). PANAMá: Bocas del Toro: SE Almirante, across bay, S. McDaniel 5157 (MO); vicinity of Chiriquí Lagoon, H. von Wedel
1365 (MO). Canal Zone: Vicinity of Mindi, P. H. Allen 5119 (MO, NA); Fort Sherman on Peña road, E. L. Tyson y R. Ah Chu 1692 (DUKE). Colombia: Amazonas: Trapecio Amazónico, entre los ríos Loretoyacu y Hamacayaca, $J$. M. Duque-Jaramillo 2386 (COL); misma localidad, R. E. Schultes y G. A. Black 8522 (COL). Antioquía: Carase, Bro. Daniel 2063 (US). Vaupés: orilla del Río Guaviare al otro lado de San Fernando, J. Aranque Molina y F. Barkley 218 (COL). venezuela: Apure: Pedro Camejo, banks of the Río Meta, near Brazo La Martinera, 19.5 airline km W of Mata de Guanábano, G. Davise y A. González 14036 (MEXU, MO); Distr. Pedro Camejo, banks of the Río Orinoco, opposite Isla Pantallo, G. Davidse y A. González 14493 (MEXU, MO). Bolívar: Cd. Bolívar and vicinity of the Orinoco, Río Buena Vista, L. H. Bailey y E. Z. Bailey 1797 (US). GUYANA: Cuyuni-Mazaruni Region: Essequibo River, between Omai and Dennison Mine Camp, near Kumaka, L. J. Gillospie 1440 (MEXU, US). PERú: Loreto: Quebrada Tahuayo, above Tamishiyaco, T. B. Croat 19741 (MEXU, MO); Maynes, Indiana, Quebrada Yanayacu, S. McDaniel 22286 (DUKE, MEXU); Atún Cocha (Río Saniria), R. Vásquez y N. Jaramillo 6470 (MEXU, MO). Bolivia: Vaca Diez: Beni, ca. $5 \mathrm{~km} \mathrm{SW}$ of Riberalta, J. C. Solomon 7927 (MEXU, MO). BRASIL: Acre: Bacia do Acre, Rios Purus e Acre, Lago da Cobra, G. T. Prance et al. $48777(\mathrm{~K})$; Bôca do Acre, Rios Puros and Acre, around Lago da Cobra, Rio Purus, N bank opposite Bôca do Acre, G. T. Prance et al. 2418 (COL); Amazônas: Sâo Paulo de Olivença, A. Ducke 563 (US); Rio Solimôes, Lago Buiussú, M. Silva 2014, 2016 (NY). Pará: S forest of the I. A. N., Belén, W. A. Archer 7953 (US). Roraima: Mpio de Boa Vista, Reserva Ecológica de Maracá, Rio Urariquera, G. P. Lewis 1429 (K, E); Canto Galo, S bank of Rio Mucajai, between Pratinha and Rio Apias, G. T. Prance et al. 4083 (K).

\section{Agradecimientos}

A Fernando Chiang Cabrera por sus sugerencias nomenclaturales y su traducción al latín de la diagnosis; a Gloria Andrade por organizar el manuscrito e incorporar la información; a Ramiro Cruz Durán por su magnífica lámina botánica; a los curadores de los herbarios BM, COL, DS, DUKE, E, F, GH, K, MEXU, MO, NA, NY, $\mathrm{P}, \mathrm{US}, \mathrm{W}$ por facilitar los ejemplares para su consulta, en préstamo o en visitas a las instituciones.

\section{Literatura citada}

Aublet, J. B. C. F., 1775. Histoire des plantes de la Guiane Françoise 2 :750. Catalogue des libres nouveaux. Paris. Azevedo-Tozzi, A. M. G., 1989. Estudos taxonômicos dos 
gêneros Lonchocarpus Kunth e Deguelia Aubl. no Brasil. Tesis doctorado, Universidade Estadual de Campinas, Campinas, Brasil. 341 p.

Azevedo-Tozzi, A. M. G., 1994. Especies noves de Deguelia Aubl. (Leguminosae- Papilionoideae-Millettieae). Revista Brasileira de Botânica 17: 45-52.

Azevedo-Tozzi, A. M. G, 1998. A identidade do timbóvedadeiro: Deguelia utilis (A. C. Sm.) A. M. G. Azevedo (Leguminosae-Papilionoideae). Revista Brasileira de Biologia 58:511-516.

Bentham, G. 1860. Synopsis of Dalbergieae, a Tribe of Leguminosae. Journal Linnean Society Botany 4 (Supplement): 1-128.
Geesink, R. 1984. Scala Millettieaerum. A survey of the genera of the tribe Millettieae (Leguminosae-Papilionoideae), with methodological considerations. Leiden Botanical Series 8 . E. J. Brill/Leiden University Press, Leiden.

Mattos, N. F. 1988. O género Derris Lour. no Rio Grande do Sul. Loefgrenia 93: 1-14.

Pittier, H. 1917. The Middle American species of Lonchocarpus. Contributions from the United States National Herbarium 20:37-93.

Poppendieck, H. H., 1999. 22 Derris Lour. (Fabaceae). In Flora of the Venezuelan Guayana 5, P.E. Berry, K. Yatskievych y y B. K. Holst (eds.). Missouri Botanical Garden Press, St. Louis. p.302-304. 